\title{
D'UNE PRATIQUE À CONTRE-TEMPS À UNE ACTIVITÉ À CONTRE-ESPACE* Une interprétation géographique de l'émergencedu wild-boaring en Pyrénées françaises
}

\author{
André Suchet \\ Institut de géographie alpine, Université de Grenoble/CNRS, France \\ a.suchet@wanadoo.fr
}

\begin{abstract}
Résumé: La pratique du canyoning, représente une composante importante de l'activité sportive et du tourisme de montagne dans les Pyrénées, aussi bien en France qu'en Espagne. Après avoir étudié démographiquement les différentes modalités de la pratique du canyoning dans le sud de la France, il est apparu qu'un certain nombre d'individus se retrouvait pour descendre les canyons "le soir", notamment "après le travail". Il s'agit en particulier de fuir la fréquentation et les groupes de touristes avec leur moniteur. Par suite, une seconde étude réalisée dans le massif du Canigou, en Pyrénées françaises, révèle au sein de ces groupes, l'émergence d'une pratique spécifique sous le nom de wildboaring. Le wild-boaring consiste à descendre des canyons encombrés, buissonnants et relativement peu intéressants selon les critères habituels de l'activité canyoning.

Cette transformation marque, selon nous, le passage d'une pratique à contre-temps à une activité à contre-espace. Par cet aspect, se joue l'exacerbation d'une forme nouvelle de rejet du système économique marchand au sein des pratiques de nature et une forme particulière de relation sociale à l'espace. L'article discute également la possibilité d'une élaboration géographique de contre-lieux par l'activité, mais cette hypothèse n'est pas validée.
\end{abstract}

Mots-clés: canyoning, wild-boaring, Pyrénées, contre-temps, contreespace, tourisme de montagne.

Abstract: The practice of canyoning represents an important part of mountain tourism in the Pyrenees, as well as in France and in Spain.

* Recibido: 13-04-10. Aceptado: 24-11-10. 
After studying demographically different modalities of canyoning in southern France, it is apparent that a number of individuals descend canyons "in the evening", including "after work". This is especially in order to avoid tourist groups with their guide. As a result, a second study in Canigou in the French Pyrenees reveals within these groups, the emergence of a specific activity under the name of wild-boaring. The wild-boaring involves crowding down, bushy and relatively uninteresting canyons as usual criteria of canyoning.

This transformation marks, we believe, the passage of a practical counter-time into counter-space activity. This aspect displays an exacerbation of a new form of rejection of the economic system in commercial outdoor recreation and of a particular form of social relationship to space. The paper also discusses the possibility of couter-places produced by the activity, but this hypothesis is not validated.

Keywords: canyoning, wild-boaring, Pyrenees, counter-time, counterspace, mountain tourism.

Activité hybride de randonnée, d'escalade, de spéléologie et de nage en eau vive, le canyoning, ou barranquismo en Espagnol, consiste en la descente à pied et à la nage d'un cours d'eau encaissé appelé canyon comportant ressauts, cascades et toboggans naturels. Inventé dans les Pyrénées française et espagnoles au tournant des années 1970 et 1980 (Suchet, 2008: 3-5), l'activité canyoning s'inscrit dans le mouvement des nouveaux loisirs sportifs de montagne et de nature (Dienot \& Theiller, 1999; Loret, 1995). Cette activité connaît depuis le début des années quatre-vingt un important développement attribuable notamment "à son caractère très ludique, qui privilégie un plaisir immédiat” (Bourdeau, 1994: 50). En France, localement, des études par recoupement indiquent 11000 passages annuels pour 9500 usagers dans le département des Pyrénées-Atlantiques (DRDJS Aquitaine-Gironde, 2006: 9) et environ 30000 passages annuels en Pyrénées-Orientales (DDJS Pyrénées-Orientales, 2008: 2). En Espagne, sur le versant sud des Pyrénées, la Sierra de Guara est devenue un haut lieu de l'activité pour l'esthétique de ses vasques d'eau turquoise serties de roches ruiniformes. Le canyoning y représente un secteur économique important dans le cadre d'un tourisme de montagne international (Theiller \& Audeguis, 2008).

En fait, à partir d'une étude socio-démographique réalisée dans le sud de la France, Suchet \& Jorand (2009) identifient trois principaux types d'usagers: les personnes encadrées par un professionnel de l'activité, ${ }^{1}$ souvent des vacanciers qui ne

\footnotetext{
${ }^{1}$ A leur sujet, Suchet \& Jorand (2009) montrent que le taux de passage d'une activité encadrée payante à une modalité autonome est inférieur à $5 \%$. Ces personnes déclarent lors d'entretiens complémentaires vouloir se tourner vers d'autres activités et s'inscrivent dans la culture des vacances polyvalentes plutôt haut de gamme (sportives, festives mais aussi culturelles avec la visite d'un musée ou d'un lieu d'histoire).
} 
pratiqueront qu'une seule fois l'activité "pour découvrir" (67\% de la fréquentation), les pratiquants réguliers en club ou en famille ${ }^{2}$ dont le niveau technique "permet de se faire plaisir sans toutefois prendre des risques" (16\% de la fréquentation) et enfin, les "passionnés" ainsi qu'ils se nomment, qui ne représentent que $8 \%$ de la fréquentation mais dont le profil est bien particulier.

Il s'agit d'hommes âgés quasiment tous de plus de 40 ans, et pour plusieurs d'entre eux, plus de 60 ans. Ils sont résidants sur le département en zone rurale, ces "passionnés", ainsi qu'ils se définissent, pratiquent toute l'année, c'est-à-dire aussi en conditions difficiles: canyons partiellement gelés l'hiver, encombrés après les crues au printemps ou à l'inverse complètement secs l'été. Issus des activités de montagne (escalade, alpinisme) ou des sports sous-marins et de la spéléologie, ces pratiquants considèrent le canyoning comme une activité physique à part entière, mais contestent le qualificatif de "sport" ainsi que le vocable "canyoning" au profit de "canyon" jugé moins "commercial". Attachés à la notion de milieu naturel "grandiose", pour eux: "en canyon, on ne rigole pas". Ils sont souvent les ouvreurs et premiers équipeurs de parcours, réalisent des voyages consacrés à l'activité (Sierra de Guara, Andalousie...) et disposent d'une importante connaissance du milieu. Ils voyagent en dehors des périodes de vacances, et tentent "d'éviter les week-ends". Techniquement, ils préfèrent les franchissements en libre (réalisation de sauts à plus de 20 mètres de hauteur, toboggans difficiles, désescalades exposées...). En l'absence d'une structure familiale forte (divorce, séparation...), ils s'investissent grandement dans l'activité: "depuis mon divorce, je ne fais plus que du canyon". Retraités, sans emploi, techniciens ou employés, ils s'inscrivent socialement dans les classes populaires. Parfois en club (principalement le CAF), ils refusent l'engagement administratif, critiquent les "excès de réglementation" et déclarent rechercher "l'évasion", l'ailleurs.

Ces "passionnés" totalisent de 40 à 100 sorties année, à des horaires parfois particuliers (le soir "après le travail", la nuit...), les raisons évoquées sont alors: "éviter la fréquentation" ou "la magie des lieux". Cette pratique de l'activité canyoning se caractérisent par un usage des sites à contre-temps. "En semaine", "en dehors des périodes de vacances", le soir, la nuit... s'oppose au temps de pratique socialement dominant. Dans l'intention de rejeter la fréquentation touristique, les "passionnés de canyon" inventent une pratique à contre-temps de l'activité canyoning (fig. 1).

A la suite de ce constat (Suchet \& Jorand, 2009), il est apparu qu'un certain nombre d'individus se retrouvaient pour descendre les canyons à sec ou en eau crou-

\footnotetext{
${ }^{2}$ Cette seconde catégorie regroupe les pratiquants autonomes, dits aussi pratiquants indépendants, c'està-dire qui pratiquent l'activité en dehors d'un guide ou d'un moniteur de canyoning. Par contre, les regroupements associatifs sont nombreux. Souvent, les personnes pratiquent également d'autres activités de montagne et de nature (randonnée, escalade, alpinisme, spéléologie ou canoë-kayak).
} 


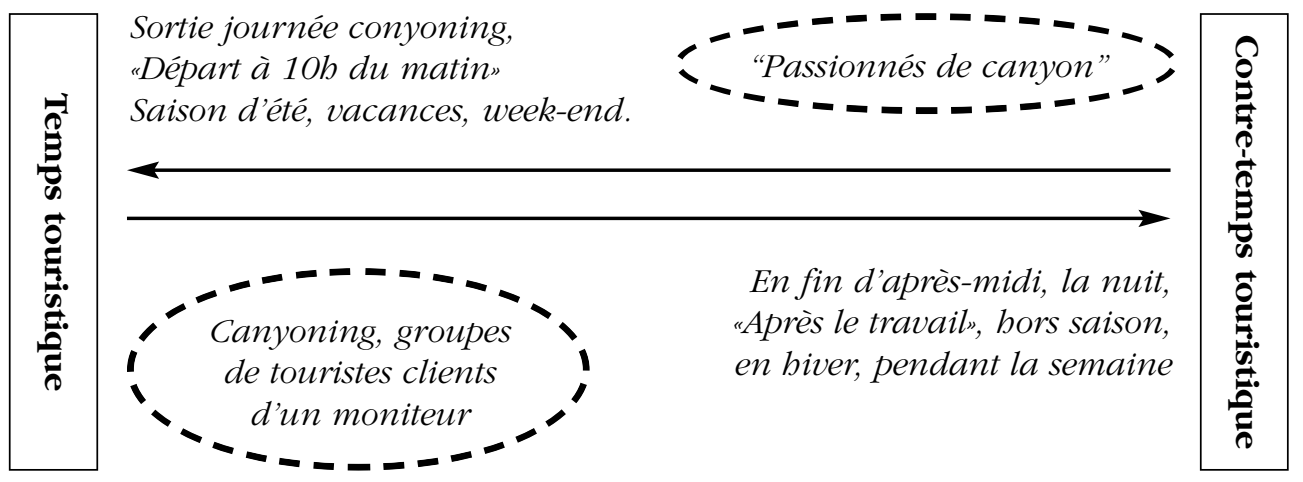

Figure 1. La pratique des «passionnés de canyon» en tant que contre-temps-touristique.

pissante, encombrés de branches, de ronces, buissonnants et relativement peu intéressants selon les critères habituels de l'activité canyoning. Des parcours ou l'on ne peut pas sauter dans l'eau ni glisser. Une pratique développée quasiment séparément de l'activité canyoning sous le nom de wild-boaring ou carabouze. Selon nous, cette transformation marque le passage d'une pratique à contre-temps vers une activité à contre-espace. Le présent article vise à comprendre les motivations géographiques, sociales ou (contre)économiques de cette évolution dans l'existence de terrain des pratiques de wild-boaring en Pyrénées françaises.

Sur un plan méthodologique, cet article fait suite à plusieurs phases d'observations participantes en wild-boaring durant les étés 2007 et 2008. Le terrain d'étude se situe en Pyrénées orientales autour des villes de Prades et de Céret. À chaque fois des entretiens étaient réalisés avant, pendant et après l'activité. Démarche géographique et ethnographique. Conjointement, des sources écrites, principalement des textes mis en lignes ont été utilisées. Dans le traitement par analyse catégorielle thématique (Bardin, 2003), la grille d'analyse concernait: définition de l'activité, relation à l'environnement, définition de l'environnement, masculinité/féminité, risque/sécurité, sources de plaisir, techniques mises en œuvre, idéal de pratique/pratique rejetée/pratique passée/pratique réelle, et plus spécifiquement: relation au temps et à l'espace touristique/non touristique.

\section{L'émergence du wild-boaring}

A propos du vocable wild-boaring, des relevés de discussion dans un forum Internet ${ }^{3}$ affirment "le wild-boaring est une variante du canyoning, dont la caracté-

${ }^{3}$ Consulter en particulier les forums du site: http://www.descente-canyon.com/ 
ristique principale est de se dérouler dans un cadre sauvage et inhospitalier. Cette pratique est apparue depuis quelques années en Europe dans la chaîne des Pyrénées, notamment dans les régions Est du massif où le relief s'abaisse et se fragmente. Plusieurs groupes fréquentent ainsi le massif du Canigou, le Vallespir et les Albères à la recherche de possibilités inexplorées. Quelques pratiquants résident également dans les Alpes du Sud autour de Marseille et tentent des équipées à l'étranger, ${ }^{4}$ mais l'essentiel reste centré dans les Pyrénées. En premier lieu, les tenants du wild-boaring affirment un attrait pour la "sauvagerie" par opposition aux espaces "domestiqués" (Parlebas, 1999: 108-113), comme si les parcours faciles d'accès ne suffisaient plus à satisfaire leur besoin de "pleine nature” (Marty, 1988). Plus précisément, le wild-boaring consiste à descendre des canyons encombrés, buissonnants et relativement peu intéressants selon les critères habituels de l'activité.

\subsection{Au delà d'un contre-temps}

Au départ, le wild-boaring participe au mouvement d'affirmation d'une pratiquecanyon tout au long de l'année, c'est à une pratique hors-saison. Effectivement, pour les puristes de l'activité, la descente de canyon ne doit pas être considérée comme une pratique saisonnière. Ces derniers rejettent fortement les discours assimilant le canyoning à une sorte "d'animation de vacances". Dans cet objectif, les passionnés multiplient les signes d'activité durant l'hiver (rassemblements hivernaux, ${ }^{5}$ progression dans des parcours gelés avec escalade des cascades de glace, descente de canyon dans des sources sulfureuses d'eau chaude...) et concernant le wild-boaring l'article de l'encyclopédie Wikipédia affirme: "Cette activité peut constituer une alternative [...] pendant les périodes de pluie, car les canyons classiques deviennent impraticables avec l'augmentation du débit.. ${ }^{6}$ Dans le même sens, une personne que nous avons rencontré explique "dans ce type de parcours on se mouille pas trop, donc en saison froide....' Des canyons secs ou quasiment secs peuvent être descendus au printemps.

\footnotetext{
${ }^{4} \mathrm{http}: / /$ www.descente-canyon.com/canyon.php/901/251/page.html

${ }^{5}$ Les rassemblements hivernaux qui se développent, -en particulier Canyon on Ice: Rassemblement international hivernal de descente de canyon organisé depuis 2008 sur la frontière pyrénéenne entre la France et l'Espagne- reçoivent progressivement le soutien financier d'entreprises internationales, notamment les fabricants de matériel Five Ten, Petzl, Résurgence... La pratique semble ainsi encore rattrapée par le monde économique.

${ }^{6}$ Anonyme, Wild-boaring, contribution à l'encyclopédie en ligne Wikipedia, page consultée le 10/03/08, http://fr.wikipedia.org/wiki/Wild-boaring. Nous ne sommes pas l'auteur de la page concernée.

${ }^{7}$ Philippe, entretien 2007.
} 


\subsection{Une pratique à contre-espace}

Au-delà d'une recherche de contre-temps, le wild-boaring se compose d'une relation forte à l'inconnu. Selon ses adeptes "le wild-boaring découle essentiellement de l'aspect exploratoire inhérent au canyonisme, qui s'est rapidement trouvé étouffé, dans les pays occidentaux, par la colonisation systématique de la plupart des grands canyons". Il s'agit de "laisser les touristes de côté", mais aussi de "découvrir de nouveaux parcours" par "envie". Dans les témoignages, une catégorisation thématique transversale (Bardin, 2003) donne raison à l'imaginaire de Prométhée "le rocher constitue un défi, provoque au viol [...]. La motivation essentielle est alors l'agression et l'ascension se résume ici à l'effort" (Bozonnet, 1992: 42). Plusieurs adeptes du wild-boaring affirment ainsi sans ambiguïté: "c'est sûr que c'est pénible, c'est pas agréable les ronces, mais si tu veux, y'a une sorte de plaisir à explorer dans la végétation [...] t'y vas à la scie [...] mais c'est la soif de première". "Une fois qu'on est passé, à la limite, c'est plus vraiment intéressant ${ }^{9} .{ }^{9}$ A plusieurs reprises, les discussions se réfèrent aux fantasmes de domination sexuelle du milieu naturel et la descente de canyon se trouve redéfinie comme un indicateur de masculinité (Connell, 1995). Par opposition, les groupes de clients avec leur moniteur sont accusés de féminité: "quelque part tu violes le canyon, cette petite gorge elle a jamais vu personne passer, t'imagines!”. ${ }^{10}$ "Quelque part, le mono avec son petit groupe qui glisse sur les toboggans ... c'est une femme le type! C'est passif. Les gens, ils font du canyon comme des femmes". ${ }^{11}$ Par cet aspect le wild-boaring correspond directement à une recherche de virginité, de conquête et de domination caractéristique de l'alpinisme européen du XIX ${ }^{\text {eme }}$ siècle (Corneloup, 1995; Hansen, 2000: 21-23; Venayre, 2002), puis de la spéléologie exploratoire au XX ${ }^{\text {eme }}$ siècle en France (Schut, 2007). En alpinisme, on pense notamment aux expressions qui assimilent la montagne à un objet de désir et de domination: “Il y a, par le monde, des mâles, qui laisseraient tomber toutes les femelles, et donneraient bien des femmes pour se coucher sur ce pan de granit et le serrer entre leurs cuisses". ${ }^{12}$ "La montagne était une belle fille, qui s'offrait à lui, sans hésiter, qui pour lui se découvrait, se dénudait...". ${ }^{13}$ "La douleur elle-même comme une forme particulièrement délicate de volupté [...] elle donne des joies insoupçonnables". "Chaque fois que j'ai fait une première, je me suis dit: te voilà plus fort». ${ }^{14}$ En spéléologie, les ouvrages du siècle passé sont tout aussi explicites: "La principale raison qui nous fait aimer Ila spéléologie] c'est l'attrait de la découverte. Quelle sensation exaltante, sensuelle, quasi-érotique

\footnotetext{
${ }^{8}$ Anonyme, art. cit.

${ }^{9}$ Entretiens avec un groupe en wild-boaring.

${ }^{10}$ Jo, pratiquant depuis 1990 actuellement âgé de 69 ans, entretien 2007.

${ }_{11}$ Philippe, pratiquant depuis 1975, entretien 2007.

12 Max Aldebert, 1950, Le royaume des hautes terres. Paris, Durel. p. 65.

${ }^{13}$ Jean Château, 1963, Le chemin de Clarabide. Paris, Arthaud. p. 198.

${ }^{14}$ Eugen Guido Lammer, 1931, Fontaine de Jouvence: ascensions et réflexions d'un alpiniste solitaire. Chamonix: Landru. p 102 et 104. Première édition en allemand, 1891.
} 
de déflorer une caverne !’.15 Sur le terrain, sans que ce soit explicitement l'objectif annoncé, le wild-boaring participe à une forme d'élitisme clairement identifiable. Il ne s'agit pas d'un élitisme social, scientifique ou financier, mais plutôt d'un élitisme communautaire (les sorties sont organisées uniquement entre connaisseurs) et phallocratique (le groupe que nous avons pu observer se composait exclusivement d'hommes entre 40 et 70 ans). Effectivement, si l'on y prête attention, un nombre très important de plaisanteries concerne directement les organes masculins et, d'autre part, l'observation d'une fin de journée en wild-boaring montre que le moment où les hommes se changent, c'est-à-dire quittent leur combinaison néoprène mouillée pour enfiler des vêtements de randonnée puis rejoindre les voitures en bas de la vallée, constitue des moments d'exhibition, ${ }^{16}$ avec des mises en scène de la nudité masculine parfois caricaturales.

Pendant notre étude, un certain nombre de questions portait également sur un aménagement touristique dénommé Canyoning Park que nous avions étudié antérieurement (Suchet \& Jorand, 2007, voir également Bartczak, 2004; Bartczak, Charlot, \& Barnier, 2004). ${ }^{17}$ Contrairement aux attentes d'un rejet de cet "Aqualand d'un nouveau genre", les plus investis en descente de canyon expliquent "on est très content de Canyoning Park", "Canyoning Park, je suis pour! C'est bien qu'ils aient fait ça, surtout au milieu de la fête foraine, avec Argelès loisir et tout ça...”. La position de ces pratiquants de canyon et tout particulièrement de wild-boaring est intéressante. Leur justification met en évidence leur souhait de voir se concentrer la fréquentation touristique dans cet espace, pour en éviter l'investissement en sites naturels. "Canyoning Park, comme ça, tout le tourisme, les groupes en vacances, ca va aller sur canyoning Park, c'est bien ça, viendra moins polluer les sites en montagne". "Tous ces touristes en vacances qui font une 'sortie canyoning', ils devraient tous rester dans Canyoning Park,. ${ }^{18}$ Sur le terrain, l'activité wild-boaring représente ainsi l'investissement d'un contre-espace touristique, dit aussi espace contre-touristique (fig. 2).

\footnotetext{
${ }^{15}$ Jean Cadoux, Jean Lavigne, Géo Mathieu et Louis Potie, 1993, Opération -1000. Marseille, Laffitte. pp. 102-103. Première édition en 1955.

${ }^{16}$ Nous avions déjà observé la même situation parmi les groupes de passionnés en canyoning (Suchet, 2009).

${ }^{17}$ Ouvert depuis 2003 au cour de l'espace loisir d'Argelès-sur-Mer (dans le département des PyrénéesOrientales) entre un site d'attraction foraine, un karting, un bowling et un parc acrobranche, Canyoning Park comporte une dizaine de sauts, autant de toboggans, deux descentes en rappel et une tyrolienne de cent mètres en fin de parcours. Composée d'un échafaudage métallique recouvert de béton projeté pour offrir l'illusion du rocher, la structure est aménagée en demi-cercle autour d'une avancée surélevée du complexe-bar. La fréquentation (des petits groupes équipés d'un matériel collectif standard sur un circuit défini pour un prix forfaitaire de $29 €$ pour les adultes et $25 €$ pour les enfants) se compose de vacanciers suivant le principe et les motivations d'un parc de loisir.

${ }^{18}$ Extraits d'entretiens avec des pratiquants de wild-boaring.
} 


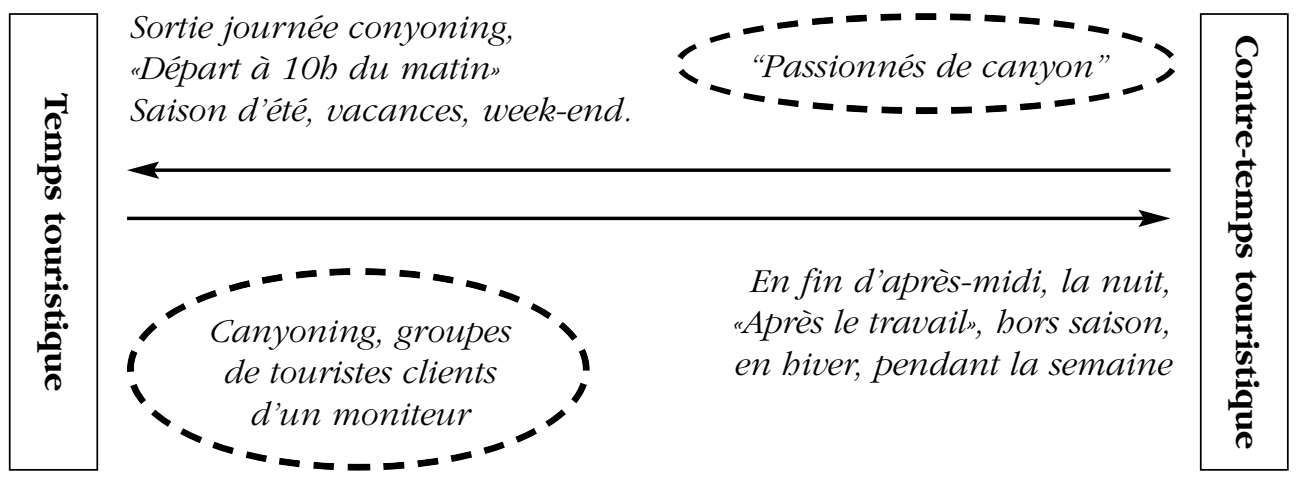

Figure 2. L'activité wild-boaring en tant qu'investissement d'un contre-espace touristique, dit aussi espace contre-touristique.

\section{Une forme originale de rejet du système économique marchand}

Le wild-boaring participe à une forme nouvelle de rejet du système économique marchand au sein des pratiques de nature. Un rejet de l'économie marchande, par delà une situation de dénégation (Aubel \& Ohl, 2004). Il s'agit de prendre à contrepied la marchandisation engendrée par l'intégration des activités de nature initialement conçues dans une logique alternative (Loret, 1995) au sein de l'industrie des loisirs et du tourisme de nature (Dienot \& Theiller, 1992; Perrin \& Mounet, 2004). Effectivement, la littérature sociologique en France ou au plan international (Lacroix, 1988; Nasser, 1996; Wheaton, 2000; 2004; Weed, 2008) montre bien comment ces activités de nature, surf, windsurf, deltaplane, parapente, escalade, rafting... sont paradoxalement devenues un secteur commercial en magazines, vêtements de mode, matériel technique ou destinations de voyage. En France, le canyoning, avec seulement $24 \%$ de la fréquentation des sites composée de pratiquants indépendants, contre $67 \%$ de clients d'une structure commerciale (Suchet \& Jorand, 2009), représente d'ailleurs l'une des activités particulièrement illustrative de ce phénomène. Identiquement, el barranquismo en Espagne constitue l'un des secteurs économiques majeurs de certaines communes, notamment dans la Sierra de Guara (Theiller \& Audeguis, 2008).

Dès lors, considérant la facilité du canyoning (Bourdeau, 1994) comme responsable de sa marchandisation, les tenants du wild-boaring s'engagent dans des parcours extrêmement difficiles d'accès et/ou comportant des passages particulièrement délicats: canyons peu irrigués envahis par la végétation, c'est-à-dire encombrés de pierres, de ronces, de troncs d'arbres coincés au dessus des rares vasques d'eau crou- 
pissante... Dans une sorte de retour en arrière, vers l'alpinisme hard et la pénibilité de la nature à vaincre (Bozonnet, 1992), les personnes taillent les branches, franchissent des talus d'éboulis et passent dans la boue. ${ }^{19}$ Pour ces pratiquants, le wild-boaring constitue ainsi "l'antithèse de l'image commerciale du canyonisme vébiculée par les médias: sauts et toboggans dans une eau limpide, ruisselante dans un pur cadre rocheux ensoleillé..... ${ }^{20}$ Le rejet des groupes de clients avec leur moniteur se trouve alors effectivement satisfait, puisque la pénibilité des sites qualifiés de wild-boaring n'intéresse pas les structures commerciales qui ne fréquentent pas ces parcours.

En fait, le terme wild-boaring correspond à une traduction par des non-anglophones du mot "sanglier", ce type de canyon étant surnommé "canyon à sanglier" ou carabouze. Autrement dit, pour les adeptes de cette éthique de la descente de canyon, l'invention d'un terme anglophone "ridicule" signifie une dérision vis-à-vis de la consonance attractive "des sports ...en "ing”. Encore une preuve du rejet de l'espacetemps et des normes de l'économie marchande, matérialisée en montagne par la sphère du tourisme. Autant d'éléments qui rappellent les résultats de Léséleuc dans le cas du site d'escalade de Claret (de Léséleuc, 1998; 2004; de Léséleuc et al., 2002). Sinon, carabouze vient de la contraction de caracal et de bouse. Le premier est un animal solitaire d'Afrique et d'Asie, petit félin proche du lynx. Le second renvoie aux excréments des bovidés. "Une carabouze est donc un canyon à l'intérêt limité difficile d'accès, inconnu". ${ }^{21}$ Géographiquement, la référence à un animal solitaire, et cette intention contre-marchande vient des Pyrénées orientales et des Alpes du Sud, c'està-dire en France, les aires le plus fréquenté commercialement par des groupes avec leurs moniteurs, comme une sorte de réaction locale.

\section{Le wild-boaring comme fabrique de contre-lieux?}

Conceptuellement, au delà du fait que les activités sportives de nature en montagne constituent un ailleurs récréatif, par opposition au monde quotidien urbanisé (Bourdeau, 2003), les sites de wild-boaring peuvent apparaitre en tant que contrelieux de société. En tout cas, c'est une hypothèse géographique possible dans l'étude de cette émergence praxique. La notion de contre-lieu développée dans le cas des activités de nature en montagne par Cailly (2006) provient notamment d'une étude remarquable de Léséleuc dans le sud de la France. Cet auteur traite d'un petit groupe de grimpeurs ayant produit une organisation sociale de type néo-communautaire en un site d'escalade, la falaise de Claret proche de Montpellier (de Léséleuc, 2004; de

\footnotetext{
${ }^{19}$ Christian, pratiquant depuis 1998, entretien 2007; Michel, pratiquant depuis 1980, entretien 2008

${ }^{20}$ Anonyme, Wild-boaring... art. cit.

${ }^{21}$ http://www.descente-canyon.com/canyon.php/901/251/page.html
} 
Léséleuc et al., 2002). L'auteur y observe notamment un rejet de la rationalité économique (de Léséleuc, 1998), c'est-à-dire du tourisme ou de la fréquentation consumériste de ceux qualifiés "d'étrangers", par ceux auto-qualifiés de "locaux".

Néanmoins, il ne semble pas possible d'observer dans les équipées de wild-boaring la fabrication de contre-lieux, dans la mesure où l'espace du wild-boaring, ces carabouzes, ne sont pas appropriés. Il n'y a ni lieux ni contre-lieux. A la différence du site d'escalade de Claret étudié par de Léséleuc (2004), aucune démarche de territorialisation n'est mise en ouvre, aucun marqueur territorial, ni processus de patrimonialisation (Di Méo, 1994, 1998) ne transparait. Par exemple, l'équipement des sites de wild-boaring n'est pas voulue, au contraire "on essaye de laisser un minimum de matériel, ...parce que, ...parce que ça coute, si on laisse des sangles et tout dans les arbres... tu perds du matos". ${ }^{22}$ Autre exemple, dans les pratiques de canyoning à contre-temps, les passionnés indiquent parfois l'entrée du site par un cairn, c'est-àdire l'une des premières formes d'appropriation de l'espace, mais ce type de marquage ne semble pas exister en wild-boaring. Les pratiquants ne formulent pas de cartes, de topoguides ou même de pages internet pour attester leurs découvertes.

La territorialisation étant précisément l'ensemble des processus par lesquels un individu ou plus souvent un groupe social s'approprie un espace en lui imposant une configuration matérielle et symbolique qui constitue un support actif de son activité identitaire (Cailly, 2006; Di Méo, 1998). L'intention phallocratique et contre-marchande trouve ici un terrain d'exercice, mais celui-ci reste la manifestation spatiale d'une intention sociale.

\section{Conclusion}

La pratique du canyoning, représente une composante importante de l'activité sportive et du tourisme de montagne dans les Pyrénées (Dienot \& Theiller, 1992; Perrin \& Mounet, 2004; Suchet \& Jorand, 2009; Theiller \& Audeguis, 2008), aussi en bien en France qu'en Espagne. Une variante de l'activité apparue depuis quelques années en Pyrénées françaises sous le nom de wild-boaring consiste à descendre des canyons encombrés, buissonnants et relativement peu intéressants selon les critères habituels de l'activité canyoning. Dans le discours des personnes, il s'agit d'éviter la fréquentation des groupes de touristes avec leur moniteur. Le wild-boaring provient effectivement de quelques "passionnés de canyon" qui évitaient déjà la fréquentation commerciale en se retrouvant pour descendre des canyons à des horaires particuliers : en fin de journée, la nuit... Cette transformation marque, selon nous, le passage d'une

${ }^{22}$ Entretiens avec un pratiquant de wild-boaring. 
pratique à contre-temps à une activité à contre-espace. Par cet aspect, se joue l'exacerbation d'une forme nouvelle de rejet du système économique marchand au sein des pratiques de nature et une forme particulière de relation sociale à l'espace. Mais au delà d'un rejet du tourisme, les motivations concernent également une recherche de virginité et de conquête du milieu naturel en phase avec une masculinité précisément affichée. Par contre, l'hypothèse d'une élaboration de contre-lieux tels que décrits dans la littérature (Cailly, 2006; de Léséleuc, 2004), ne tient pas. Quasiment aucune forme de territorialisation de l'espace ne transparait. Le wild-boaring reste seulement la manifestation spatiale d'une intention sociale.

Cette variante du canyoning ne concerne pour le moment qu'un nombre restreint de pratiquants dans une aire géographique des Pyrénées françaises très circonscrite. Le wild-boaring n'est apparu que très récemment en d'autres espaces : dans les Alpes du Sud ou à l'étranger. L'intention n'est pas de contrer l'activité canyoning. Néanmoins, au delà d'un cas singulier de rejet du système économique marchand particulièrement intéressant, il s'agit d'observations à ne pas négliger dans le sens où elles risquent de s'inviter dans le métissage producteur des activités sportives en montagne de demain.

\section{Bibliographie}

Aubel, O., \& Ohl, F. (2004): The denegation of the economy, The Example of Climbing in France. International Review for the Sociology of Sport, 39(2), 123-137.

Bardin, L. (2003): L'analyse de contenu. Paris, Presses universitaires de France.

Bartczak, F. (2004): Le Canyoning Park: un espace entre-deux. Communication au 9th Annual Congress of the European College of Sport Science, Clermont-Ferrand, du 3 au 6 juillet.

Bartczak, F., Charlot, V., \& Barnier, M. (2004): Canyoning Park: Analyse de la clientèle "pionnière" d'une première mondiale. Cabier Espaces, 81, 90-101.

Bourdeau, P. (1994): D'eau et de rocher: le canyoning. Cabier Espaces, 35, 49-54.

Bourdeau, P. (2003): Territoires du hors-quotidien: Une géographie culturelle du rapport à l'ailleurs dans les sociétés urbaines contemporaines. Le cas du tourisme sportif de montagne et de nature. Mémoire du diplôme d'Habilitation à diriger des recherches en géographie, Université de Grenoble 1, Grenoble.

Bozonnet, J.-P. (1992): Des monts et des mythes. Grenoble, Presses universitaires de Grenoble.

Cailly, L. (2006): Lieux de "grimpe", contrelieux de société? Essai sur les formes néocommunautaires et les processus de territorialisation en ouvre dans la pratique de l'escalade. Revue de géographie alpine, 94(3), 25-44.

Connell, R. W. (1995): Masculinities. Berkeley, University of California Press.

Corneloup, J. (1995): Le masculin et le féminin en escalade. In C. Pigeassou (ed.) 
Sport, culture et tradition. Actes du colloque des 14, 15 et 16 mai 1993 à Agde, pp. 321-330.

DDJS Pyrénées-Orientales (2008): Document non publié remis à l'Agence départementale de l'ONF des Pyrénées-Orientales.

Di Méo, G. (1994): Patrimoine et territoire, une parenté conceptuelle. Espaces et sociétés, 78, 16-34.

Di Méo, G. (1998): Géographie sociale et territoires. Paris, Nathan.

Dienot, J. \& Theiller, D. (1992): Les nouvelles pratiques sportives de la montagne: un champ nouveau pour le tourisme de pleine nature. In Tourisme et environnement. Paris, La documentation Française, pp. 38-47.

Dienot, J. \& Theiller, D. (1999): Les nouveaux loisirs sportifs en montagne: les aventuriers $d u$ quotidien. Talence, Maison des Sciences de l'Homme d'Aquitaine.

DRDJS Aquitaine-Gironde (2006): Canyonisme. Etat des lieux \& perspectives en Aquitaine. Rapport non publié.

Hansen, P. H. (2000): Albert Smith, l'Alpine Club, et l'invention de l'alpinisme au milieu de l'ère Victorienne. Revue STAPS 21(51), 7-27.

Lacroix, G. (1988): Glisse, fun ...et dollars. In Actes des premières assises des APPN. Toulouse: Publication de l'Université Toulouse 3, pp. 60-74.

Léséleuc de, E. (1998): Rationalité économique, "rationalité" du don: les enjeux d'un positionnement en escalade. In C. Pigeassou (ed.) Le management au service du sport : de la conception à la décision. Actes du premier congrès de la SFMS organisé à Montpellier les 11, 12 et 13 décembre 1997, pp. 218-225.

Léséleuc de, E. (2004): Les "voleurs" de falaise.
Pessac, Maison des Sciences de l'Homme d'Aquitaine.

Léséleuc de, E., Gleyse, J. \& Marcellini, A. (2002): The Practice of Sport as Political Expression? Rock Climbing at Claret, France. International Sociology, 17(1), 7390.

Loret, A. (1995): Génération glisse. Dans l'eau, l'air, la neige... la révolution du sport des "années fun". Paris, Autrement.

Marty, J. (1988): Un modèle de personnalité: le pratiquant en activités physiques de pleine nature. In Actes des premières assises des APPN. Toulouse: Publication de l'Université Toulouse 3, pp. 208-217.

Nasser, D. (1996): Desporte y turismo activo: une reflexi $\mathrm{n}$ sociol gica. In Actas del Congreso nacional de Turismo Rural y Turismo Activo, celebrado en Àvila del 31 de marzo al 2 de abril de 1995. Valladolid: Junta de Castilla y León, pp. 481-498.

Parlebas, P. (1999): Jeux, sports et sociétés. Paris, INSEP.

Perrin, C. \& Mounet, J.-P. (2004): L'insertion de l'offre de canyoning dans l'industrie touristique: une étude comparative entre le massif des Bauges et le Vercors. Loisir et société, 27(1), 143-172.

Schut, P.-O. (2007): L'exploration souterraine. Une histoire culturelle de la spéléologie. Paris, L'Harmattan.

Suchet, A. (2008): D'eau, de roche et de lumière. Contribution à une étude des pratiques de descente de canyon. Massif des Pyrénées (France et Espagne) Montagnes Rocheuses (USA). Mémoire de Master en géographie, Université de Grenoble 1, Grenoble.

Suchet, A. (2009): Relations de genre et masculinités dans les pratiques de canyoning. In C. Ottogalli-Mazzacavallo \& J. SaintMartin (eds.) Femmes et hommes dans les 
sports de montagne. Grenoble, Maison des Sciences de l'Homme des Alpes, pp. 391408.

Suchet, A., \& Jorand, D. (2007): Les usagers des parcours naturels de canyoning et de la structure artificielle Canyoning Park (France): une approche socio-culturelle. Loisir et société, 30(2), 417-441.

Suchet, A., \& Jorand, D. (2009): La fréquentation des sites naturels de canyoning: une approche socio-culturelle. Karstologia, 53, 15-26.

Theiller, D., \& Audeguis, V. (2008): L'enjeu du développement durable des sports de nature estivaux dans la chaîne pyrénéenne. In O. Bessy (ed.) Sport, loisir, tourisme et développement durable des terri- toires. Voiron, Presses universitaires du sport, pp. 89-99.

Venayre, S. (2002): L'alpinisme: une aventure ? Remarques sur l'historicité de l'aventure. In O. Hoibian \& J. Defrance (eds.) Deux siècles d'alpinismes européens. Paris, L'Harmattan, pp. 163-174.

Weed, M. (2008): Sports Tourism Consumption and Production. Journal of Sport $\mathcal{E}$ Tourism, 13(4), 245-247.

Wheaton, B. (2000): "Just Do It": Consumption, Commitment, and Identity in the Windsurfing Subculture. Sociology of Sport Journal, 17(3), 254-274.

Wheaton, B. (ed.) (2004): Understanding lifestyle sports: consumption, identity and difference. London: Routledge. 